\title{
Stimulus-driven reorienting in the ventral frontoparietal attention network: the role of emotional content
}

\author{
David W. Frank ${ }^{1 *}$ and Dean Sabatinelli ${ }^{1,2}$ \\ Department of Psychology, University of Georgia, Athens, GA, USA \\ ${ }^{2}$ Biolmaging Research Center, University of Georgia, Athens, GA, USA
}

\section{Edited by:}

Hauke R. Heekeren, Freie

Universität Berlin, Germany

\section{Reviewed by:}

Luiz Pessoa, Brown University, USA Gilles Pourtois, University of Ghent, Belgium

\section{${ }^{*}$ Correspondence:}

David W. Frank, Department of

Psychology, University of Georgia,

Athens, GA 30602, USA.

e-mail:dwfrank@uga.edu
Activity in the human temporoparietal junction (TPJ) and inferior frontal gyrus (IFG) is hypothesized to underlie stimulus-driven, or "bottom-up" attention reorienting. Demanding tasks require focused attention, and as task difficulty increases, activity suppression in the ventral network correlates positively with task performance, an effect thought to reflect the gating of irrelevant cues. However, activation in these structures is elicited by a range of stimulus features and task demands that vary across multiple characteristics, complicating the interpretation of the functional role of this pathway. Consideration of several current studies suggests that, in addition to task difficulty, the motivational relevance or emotional intensity of distractor stimuli may supersede ongoing task priority, and evoke ventral network activation. Support for this possibility is offered from a review of recent reports, and the import of this perspective for models of attention reorienting is discussed.

Keywords: attention, emotion, fMRI, salience
The dorsal and ventral subdivisions of a frontoparietal network are persistently involved in a process of categorizing stimuli, determining the locus of attention, and disengaging and reorienting attention as necessary (Corbetta and Shulman, 2002). The dorsal branch includes regions of the intraparietal sulcus (IPS) and frontal eye fields (FEF), and tends to be active during focused, goal directed attention to a particular target. The ventral branch includes the temporoparietal junction (TPJ), inferior frontal gyrus (IFG), and anterior insula, and is associated with redirecting attention toward stimuli that are relevant to the immediate goal, regardless of low-level perceptually salient features such as color or contrast (Corbetta et al., 2008). For example, in this frontoparietal visual attention model, while searching a crowded train station for a friend wearing a white hat, the dorsal branch would show sustained activity, while the ventral branch would show transient activity each time a hat or piece of white clothing is encountered.

While the ventral reorienting network is implicated in responding to stimuli that are important to the task at hand, the range of stimulus characteristics capable of eliciting ventral network activation is loosely defined, and the operational definitions of relevance and salience differ widely across studies. Moreover, despite our highly developed human ability to focus attention, we are compelled to attend to cues that signal appetitive or aversive outcomes (Lang et al., 1997; Mogg and Bradley, 1998). Thus, there is inherent sensitivity to emotional stimuli that influence attentional reorienting independent of task-driven goals, that we consider an essential component of any functional model of the reorienting process. The potential for higher order affective stimuli to activate this ventral processing system is unknown, and may play a distinct role in attention shifts away from task relevant ("top-down") and non-emotional perceptually salient stimuli ("bottom-up").

The purpose of this brief review is to consider the range of tasks that have been shown to activate the ventral reorienting network in an attempt to integrate and refine the concept of task-relevance in this context. We separate the paradigms used to assess ventral attention network activation into sections in which we consider studies that use sensory change tasks, reorienting paradigms, or memory and virtual reality tasks. Our goal is to clarify the specific conditions in which the ventral attention network is recruited, and to highlight the potential role for emotion in our understanding of the reorienting process by reviewing recent imaging studies that we believe are particularly relevant. By incorporating results across many distinct paradigms, we hope to distill the stimulus features that elicit ventral network activation and refine how these features impact attention. Finally, we discuss future studies that may incorporate emotional and taskdirected attention processes to investigate the functional range of the ventral attention network.

\section{SENSORY AND CONTEXTUAL CHANGE}

In the absence of an ongoing behavioral task, the ventral attention network is recruited in response to simple stimulus changes across multiple modalities. Downar et al. $(2000,2001)$ presented participants with simultaneous visual, auditory, and tactile stimuli, and reported increased activity in TPJ, IFG, and insula when any of the three stimuli changed. However, when participants were asked to attend to changes in one modality while ignoring others, TPJ and anterior insula showed enhanced activation only to task-relevant sensory changes. The authors interpreted activation in the IFG as reflecting a gating function that inhibits responses to stimuli irrelevant to current goals. This is consistent with a perspective 
of the IFG as a motor inhibitory region in no-go conditions of the go/no-go task (Konishi et al., 1999). These data are clearly consistent with the hypothesized role of the ventral reorienting network as a flexible change detector that promotes the continued processing of a primary task in the face of potentially distracting peripheral stimuli (Corbetta and Shulman, 2002).

Oddball tasks are also associated with ventral network activation. A typical two-stimulus oddball paradigm involves serial presentation of frequent stimuli (standards) mixed with infrequent stimuli (targets), to which the participant responds according to task instructions (Polich, 2007). While the dorsal attention network appears to be activated by both standard and target stimuli, the ventral network is only sensitive to targets (Mantini et al., 2009). The ventral network is also responsive to deviant stimuli that are distinct from standards and targets (Marois et al., 2000; Vossel et al., 2009). This engagement by deviant stimuli in the ventral network is enhanced if stimuli characteristics are far outside the perceptual range of standards and targets, such as emotional face stimuli (Asplund et al., 2010). Thus, the ventral attention network is active in the perception of repeated task relevant stimuli, as well as in response to deviant task irrelevant stimuli. This activity profile is in line with the conception that the ventral frontoparietal network acts as an attentional "circuit breaker," such that it will redirect attention to a task irrelevant stimulus if that stimulus is sufficiently atypical (Corbetta and Shulman, 2002). The characteristics by which a task-irrelevant stimulus meets this deviance threshold is currently undefined.

\section{REORIENTING}

A significant portion of the research in visual attention explores the mechanisms of explicit reorienting. In a commonly used cueing paradigm, a subject is asked to orient attention to a centrally located predictive cue (e.g., an arrow) that informs the subject to which side of their peripheral visual field a subsequent target may appear (Posner, 1980). The central cue tends to elicit ventral network activation, due to its predictive value and thus task relevance. However, when a non-predictive cue occurring in the periphery is presented (e.g., a highlighted box surrounding the space in which a target may or may not appear), there is minimal ventral network response. In both cases, the dorsal network is recruited (Kim et al., 1999; Rosen et al., 1999).

While both dorsal and ventral attention pathways are active during target presentation, the ventral branch shows enhanced activity following invalid cues. In a recent report researchers used a modified Posner cueing paradigm, replacing arrow cues with human faces in which gaze direction predicted target location (Engell et al., 2010). Participants showed behavioral performance to invalidly cued targets consistent with the standard arrow cueing paradigm. However, unlike the arrow cueing task, the ventral network exhibited no increase in activation to targets invalidly cued by gaze direction. Despite this inconsistent finding (which may suggest distinct operating characteristics during non-standard paradigms), the majority of reorienting studies report that the ventral network is activated following a breach of expectation as a target appears in an unexpected location (Arrington et al., 2000; Corbetta et al., 2000; Hahn et al., 2006; Vossel et al., 2009). Thus, the ventral network appears to be sensitive to all target-predictive cues, and especially so in the absence of an expected presentation, during the search and following reorienting process.

The ventral network also shows limited activity to taskirrelevant stimuli that are operationally defined as salient. For example, during target search, distractor stimuli in overlapping or concurrent locations will activate dorsal but not ventral network structures (de Fockert et al., 2004; Kincade et al., 2005). These distractors do elicit attention, as shown in behavioral measures, and activate the dorsal network, but the ventral network is apparently insensitive to task-irrelevant perceptual salience as it is defined in these paradigms.

There are data to suggest that task-irrelevant stimuli defined as salient can recruit the ventral network if they share features with the target. For example, in a modified Posner cueing paradigm (Indovina and Macaluso, 2007), target-colored distractors at the unattended location elicited activity in IFG and TPJ, while no activation was evident in response to checkerboard distractors that shared few features of the colored targets. Consistent with this effect, Serences et al. (2005) report enhanced activation in the ventral network in response to flanking distractors that shared the color of the central search target. To a lesser extent, non-target colored peripheral letters also activated the ventral network, relative to gray letters. Thus, the level of ventral network recruitment may be expressed as a function of target similarity, as the target colored distractors elicited more activity than non-target colored distractors, which in turn were more activating than gray letters. These studies suggest that the ventral attention network is particularly sensitive to task relevant stimuli, regardless of distinctive perceptual characteristics.

Thus far, task-relevance has signified stimuli that are instructed targets or stimuli that share perceptual properties with an instructed target. To explore the idea of task relevance further, Natale et al. (2010) employed a peripheral reorienting paradigm that elicited TPJ activity when peripheral distractors shared the color of the target set in past trial blocks, although the current target color had changed. These data suggest that TPJ activation in this paradigm may be associated with conditioned priming by target features that gradually shift to target changes. Consistent with this, a recent study examined continuous cue stimuli that predicted the location of an upcoming target (Geng and Mangun, 2011). On selected trials, an additional colored stimulus that was partially predictive of the gray location of the targets would also be presented. This second colored stimulus shared no perceptual features with the target, but elicited activation in the ventral network. This study demonstrates that additional information that may improve task performance can activate the ventral network, despite a constant predictive cue. As this predictive information need not be perceptually similar to the target, task-relevance may represent a higher order of signal value than simple perceptual similarity.

\section{MEMORY AND VIRTUAL REALITY}

The ventral network has also been shown to be active during attention to potentially relevant stimuli for future memory retrieval. When viewing a stream of objects varying in category, the TPJ and IFG show increased activation in response to objects that belong to contextual category of the target 
(Hampshire et al., 2007). Additionally, TPJ activation appears to predict performance during target recall, showing less activity during successful relative to unsuccessful retrieval (Anticevic et al., 2010b), perhaps reflecting the relationship between TPJ and task relevance. In a recent study, authors demonstrated that the TPJ may consist of anterior and posterior subregions that are functionally and structurally separable from each other and the intraparietal lobule (Mars et al., 2011). The authors suggest that the anterior TPJ is implicated in attention processing proper, while the posterior is involved in "social mentalization" and movement (Gusnard et al., 2001). As the stimuli used in the Hampshire and Anticevic papers mentioned above lack movement and did not find TPJ activity in response to social cues, the task-similar distractors used in these studies appear to activate the anterior TPJ, though this does not preclude spatial overlap. Thus, it appears that the TPJ is involved in the process of category matching of stimuli in the visual field, according to task demands.

Researchers have constructed virtual reality environments in an attempt to approximate "real world" contexts in the lab, in order to extend the ecological validity of their results, and to enable experimental manipulations that would be impractical or impossible in reality. In one such design, participants navigated a virtual environment to a goal while avoiding unexpected obstacles in their path. In one condition, the color of a building along a familiar path abruptly changed colors. This drastic contextual change elicited TPJ activity (Iaria et al., 2008). The authors interpreted this result as support for the ventral frontoparietal network's role in detecting unexpected stimuli in real-life situations. In addition, the range of task relevance in this virtual reality context might also include another persistent task demand-harm avoidance. While a building changing color in a computer-generated environment is certainly innocuous, such a drastic context change in a realistic setting may represent significant danger.

Therefore, if one were approaching a goal, the need to ignore innocuous distractors may be measurable as an inhibition of ventral network activity, but the need to respond to potentially life-threatening events, such as a large, sudden stimulus change, would be expect to override the need for attention focus and interrupt ongoing processes to reorient and respond to the new context (Löw et al., 2008; Bradley, 2009).

In another recent study, Nardo et al. (2011) asked participants to view movies in which the environments varied with regard to peripheral distractor characteristics. In one condition, participants viewed an environment in motion, as if they were moving through it, and in another condition an unexpected virtual human appeared in the environment. Previous research has shown that the initial orienting response to the unexpected appearance of a conspecific evokes sensory intake, behavioral immobility, and preparation for action, as that conspecific may be a threat (Löw et al., 2008; Bradley, 2009). Thus, the ventral network showed enhanced activity in the unexpected human condition, demonstrating that the ventral network can be sensitive to task irrelevant stimuli in contexts in which sudden threats must be processed. Perhaps in real-world environments, and thus in human phylogeny, a critical role of the ventral network is to orchestrate task relevance with survival relevant cue processing.

\section{A ROLE FOR EMOTION IN REORIENTING}

In many research programs, a primary research interest is to describe the complex and highly developed mechanisms of attention that in some manner defines our unique human abilities. Therefore, paradigms have been developed to extract and amplify aspects of this process in a highly controlled fashion, far removed from the contexts in which this behavior evolved. If we are to understand the role of the ventral network in visual attention we may need to expand the paradigms in which it is investigated to include the range of stimuli and contexts in which the behavior evolved. Certainly the emotionality of a given stimulus plays a key role in our environmental interactions. Emotion can be thought of as a disposition toward action in which we approach appetitive and avoid aversive cues in the environment to maximize fitness (Frijda, 1986; Lang et al., 1997). Many reports demonstrate the attention-evoking nature of emotional stimuli. Examples can be drawn from a wide variety of paradigms and measures, and include the resistance of emotional words to attentional blink (Anderson, 2005), the limiting of target detection accuracy by expressive faces (Fox et al., 2001; Ohman et al., 2001; Fox, 2002; Phelps et al., 2006), the extension of gaze fixations toward emotional images (Calvo and Lang, 2004), event-related potential discrimination of emotional stimuli (Johnston et al., 1986; Naumann et al., 1992; Schupp et al., 2006; Foti et al., 2009), and differential BOLD signal during affective processing (Kensinger and Schacter, 2006; Sabatinelli et al., 2009). Emotional faces have also been shown to improve performance of target discrimination when used as primes (Pourtois et al., 2004; Brosch et al., 2011). However, only dorsal attention regions appear to be involved in reorienting (Armony and Dolan, 2002; Pourtois et al., 2006). While it is surprising that the TPJ and IFG are not involved in this process, this may reflect specialized processing of face stimuli. Faces may show unique activity, and therefore, other types of distractors such as emotional scenes may yield distinct results. In any case, affective stimuli play an important role in directing attention.

Certainly a great deal of data is well described by the frontoparietal visual attention model, in which the dorsal and ventral systems categorize stimuli, determine the locus of attention, and disengage and reorienting attention (Corbetta et al., 2008). The aim of this review was to explore the nature of stimuli that may undergo varying degrees of processing via the ventral network during focused attention. To briefly review, most studies investigating this issue have been conducted in laboratory settings using highly circumscribed paradigms. From these studies we can conclude that in the absence of a sustained focus of attention, any perceived stimulus change may increase ventral network activation. However, once the individual is engaged in a specific task, the nature of stimuli capable of redirecting attention and eliciting ventral network activity becomes restricted to those that are target-similar or target-predictive. There is also evidence for a task difficulty effect, whereby distractor stimuli are more likely to be ignored as the difficulty of the primary task increases (Anticevic et al., 2010b). Thus, during a task, perceptual distinctiveness no longer draws attention, and will activate the ventral network less so than task relevant stimuli, if at all. However, perceptually distinct items may evoke ventral network activity, if 
sufficiently potent (Iaria et al., 2008). We suggest that this potency reflects emotional intensity, such that stimuli that cue potentially threatening (or highly appetitive) outcomes may serve to interrupt ongoing processes and redirect attention, despite a top-down instruction to persevere.

It may be possible to demonstrate ventral network activity using a standard laboratory paradigm by presenting emotionally intense stimuli as task-irrelevant distractors. One study has included such a manipulation in a working-memory paradigm, yet found equivalent TPJ activity in response to aversive and neutral distractors (Anticevic et al., 2010b). In other workingmemory studies, activation in the IFG increases when subjects are exposed to arousing unpleasant images. However, unlike the TPJ, the IFG increases in activity during successful workingmemory trials when a negative distractor is present, but not in the presence of neutral distractors (Anticevic et al., 2010a; Shafer et al., 2012). Additionally, there appears to be a positive correlation between activation in the IFG and in the amygdala (Dolcos et al., 2006). These studies suggest that the IFG interfaces with the amygdala in some manner and may result in diminished distractibility during emotional picture processing. However, as these primary tasks do not require reorienting, the pattern of results is likely to be paradigmspecific.

Recent perspectives of the attentional framework suggest that perceptually salient (exogenous), goal-directed (endogenous), and emotional stimuli improve re-orienting success in an additive fashion (Brosch et al., 2011). This suggests that reorienting depends on three separate functional mechanisms. Emotional stimuli may, therefore, recruit regions in the ventral frontoparietal network; namely the TPJ and IFG. While most studies have used emotional faces to investigate this issue (Pourtois et al., 2012), other distractors such as highly arousing naturalistic scenes may

\section{REFERENCES}

Anderson, A. K. (2005). Affective influences on the attentional dynamics supporting awareness. J. Exp. Psychol. Gen. 134, 258-281.

Anticevic, A., Barch, D. M., and Repovs, G. (2010a). Resisting emotional interference: brain regions facilitating working memory performance during negative distraction. Cogn. Affect. Behav. Neurosci. 10, 159-173.

Anticevic, A., Repovs, G., Shulman, G. L., and Barch, D. M. (2010b). When less is more: TPJ and default network deactivation during encoding predicts working memory performance. Neuroimage 49, 2638-2648.

Armony, J. L., and Dolan, R. J. (2002). Modulation of spatial attention by fear-conditioned stimuli: an event-related fMRI study. Neuropsychologia 40, 817-826.

Arrington, C. M., Carr, T. H., Mayer, A. R., and Rao, S. M. (2000). Neural mechanisms of visual attention: object-based selection of a region in space. J. Cogn. Neurosci. 12, 106-117.

Asplund, C. L., Todd, J. J., Snyder, A. P., and Marois, R. (2010). A central role for the lateral prefrontal cortex in goal-directed and stimulusdriven attention. Nat. Neurosci. 13, 507-512.

Bradley, M. M. (2009). Natural selective attention: orienting and emotion. Psychophysiology 46, 1-11.

Brosch, T., Pourtois, G., Sander, D., and Vuilleumier, P. (2011). Additive effects of emotional, endogenous, and exogenous attention: behavioral and electrophysiological evidence. Neuropsychologia 49, 1779-1787.

Calvo, M. G., and Lang, P. J. (2004). Gaze patterns when looking at emotional pictures: motivationally biased attention. Motiv. Emot. 28, 221-243.

Corbetta, M., Kincade, J. M., Ollinger, J. M., McAvoy, M. P., and Shulman, G. L. (2000). Voluntary orienting is dissociated from target

also be employed, and may associated with somewhat different effects.

It will be important for future work to locate the threshold at which ecologically important distractors reorient attention away from a central task. In one such study, participants might engage in a target recognition task in which stimuli to be discriminated are presented concurrently with task irrelevant peripheral distractors. For instance, one may adapt a paradigm that is known to activate the ventral attention network via "top-down" distractor characteristics, such as used by Serences et al. (2005). This modified study would consist of a centrally attended stream of letters that is flanked by a variety of emotional or nonemotional distractor pairs. In such a design, distractor characteristics (e.g., emotional intensity, complexity) and task difficulty may be manipulated to assess the boundaries of attention reorienting. As individual emotional states have also been shown to influence attention (Fox et al., 2005, 2007; Koster et al., 2006), this may also be a useful manipulation to consider. Another potential study could involve an adaptation of the Posner cueing paradigm in which distractors consist of target-similar stimuli, abstract shapes, and emotionally arousing and neutral scenes (cf. Indovina and Macaluso, 2007). Contrasting invalid target presentations with invalid distractor stimuli, while manipulating emotional arousal and stimulus complexity may modulate ventral network activity, thus clarifying the degree to which emotional stimuli may impact this attentional process.

In summary, we propose that while the stimulus-driven ventral attention network is clearly associated with the maintenance of task focused attention, non-task related items that cue emotional relevant outcomes might have privileged access as a result of natural selection. Future work may focus on the nature of these stimuli, and investigate the interaction of emotion and attention in this ventral re-orienting process.

detection in human posterior parietal cortex. Nat. Neurosci. 3, 292-297.

Corbetta, M., Patel, G., and Shulman, G. L. (2008). The reorienting system of the human brain: from environment to theory of mind. Neuron 58, 306-324.

Corbetta, M., and Shulman, G. L. (2002). Control of goal-directed and stimulus-driven attention in the brain. Nat. Rev. Neurosci. 3, 201-215.

de Fockert, J., Rees, G., Frith, C., and Lavie, N. (2004). Neural correlates of attentional capture in visual search. J. Cogn. Neurosci. 16, 751-759.

Dolcos, F., Kragel, P., Wang, L., and McCarthy, G. (2006). Role of the inferior frontal cortex in coping with distracting emotions. Neuroreport 17, 1591.

Downar, J., Crawley, A. P., Mikulis, D. J., and Davis, K. D. (2000). A multimodal cortical network for the detection of changes in the sensory environment. Nat. Neurosci. 3, 277-283.

Downar, J., Crawley, A. P., Mikulis, D. J., and Davis, K. D. (2001). The effect of task relevance on the cortical response to changes in visual and auditory stimuli: an event-related fMRI study. Neuroimage 14, 1256-1267.

Engell, A. D., Nummenmaa, L., Oosterhof, N. N., Henson, R. N., Haxby, J. V., and Calder, A. J. (2010). Differential activation of frontoparietal attention networks by social and symbolic spatial cues. Soc. Cogn. Affect. Neurosci. 5, 432-440.

Foti, D., Hajcak, G., and Dien, J. (2009). Differentiating neural responses to emotional pictures: evidence from temporal-spatial PCA. Psychophysiology 46, 521-530.

Fox, E. (2002). Processing emotional facial expressions: the role of anxiety and awareness. Cogn. Affect. Behav. Neurosci. 2, 52-63. 
Fox, E., Russo, R., Bowles, R., and Dutton, K. (2001). Do threatening stimuli draw or hold visual attention in subclinical anxiety? J. Exp. Psychol. Gen. 130, 681-700.

Fox, E., Russo, R., and Georgiou, G. A. (2005). Anxiety modulates the degree of attentive resources required to process emotional faces. Cogn. Affect. Behav. Neurosci. 5, 396-404.

Fox, E., Mathews, A., Calder, A. J., and Yiend, J. (2007). Anxiety and sensitivity to gaze direction in emotionally expressive faces. Emotion 7 , 478.

Frijda, N. H. (1986). The Emotions. Cambridge, NY: Cambridge University Press.

Geng, J. J., and Mangun, G. R. (2011). Right temporoparietal junction activation by a salient contextual cue facilitates target discrimination. Neuroimage 54, 594-601.

Gusnard, D. A., Raichle, M. E., and Raichle, M. E. (2001). Searching for a baseline: functional imaging and the resting human brain. Nat. Rev. Neurosci. 2, 685-694.

Hahn, B., Ross, T. J., and Stein, E. A. (2006). Neuroanatomical dissociation between bottom-up and top-down processes of visuospatial selective attention. Neuroimage 32, 842-853.

Hampshire, A., Duncan, J., and Owen, A. M. (2007). Selective tuning of the blood oxygenation level-dependent response during simple target detection dissociates human frontoparietal subregions. J. Neurosci. 27, 6219-6223.

Iaria, G., Fox, C. J., Chen, J. K., Petrides, M., and Barton, J. J. S. (2008). Detection of unexpected events during spatial navigation in humans: bottom-up attentional system and neural mechanisms. Eur. J. Neurosci. 27, 1017-1025.

Indovina, I., and Macaluso, E. (2007). Dissociation of stimulus relevance and saliency factors during shifts of visuospatial attention. Cereb. Cortex 17, 1701-1711.

Johnston, V. S., Miller, D. R., and Burleson, M. H. (1986). Multiple $\mathrm{P} 3 \mathrm{~s}$ to emotional stimuli and their theoretical significance. Psychophysiology 23, 684-694.

Kensinger, E. A., and Schacter, D. L. (2006). Processing emotional pictures and words: effects of valence and arousal. Cogn. Affect. Behav. Neurosci. 6, 110-126.
Kim, Y. H., Gitelman, D. R., Nobre, A. C., Parrish, T. B., LaBar, K. S., and Mesulam, M. M. (1999). The large-scale neural network for spatial attention displays multifunctional overlap but differential asymmetry. Neuroimage 9, 269-277.

Kincade, J. M., Abrams, R. A., Astafiev, S. V., Shulman, G. L., and Corbetta, M. (2005). An event-related functional magnetic resonance imaging study of voluntary and stimulusdriven orienting of attention. J. Neurosci. 25, 4593-4604.

Konishi, S., Nakajima, K., Uchida, I., Kikyo, H., Kameyama, M., and Miyashita, Y. (1999). Common inhibitory mechanism in human inferior prefrontal cortex revealed by event-related functional MRI. Brain 122, 981-991.

Koster, E. H. W., Crombez, G., Verschuere, B., van Damme, S., and Wiersema, J. R. (2006). Components of attentional bias to threat in high trait anxiety: facilitated engagement, impaired disengagement, and attentional avoidance. Behav. Res. Ther. 44, 1757-1771.

Lang, P. J., Bradley, M. M., and Cuthbert, B. N. (1997). "Motivated attention: affect, activation, and action," in Attention and Orienting: Sensory and Motivational Processes eds P. J. Lang, R. F. Simons, and M. T. Balaban (Mahwah, NJ: Lawrence Erlbaum Associates), 97-135.

Löw, A., Lang, P. J., Smith, J. C., and Bradley, M. M. (2008). Both predator and prey: emotional arousal in threat and reward. Psychol. Sci. 19, 865-873.

Mantini, D., Corbetta, M., Perrucci, M. G., Romani, G. L., and Del Gratta, C. (2009). Large-scale brain networks account for sustained and transient activity during target detection. Neuroimage 44, 265-274.

Marois, R., Leung, H. C., and Gore, J. C. (2000). A stimulus-driven approach to object identity and location processing in the human brain. Neuron 25, 717-728.

Mars, R. B., Sallet, J., Schuffelgen, U., Jbabdi, S., Toni, I., and Rushworth, M. F. (2011). Connectivity-based subdivisions of the human right "temporoparietal junction area": evidence for different areas participating in different cortical networks. Cereb.
Cortex. doi: 10.1093/cercor/bhr268. [Epub ahead of print].

Mogg, K., and Bradley, B. P. (1998) A cognitive-motivational analysis of anxiety. Behav. Res. Ther. 36, 809-848.

Nardo, D., Santangelo, V., and Macaluso, E. (2011). Stimulusdriven orienting of visuo-spatial attention in complex dynamic environments. Neuron 69, 1015-1028.

Natale, E., Marzi, C. A., and Macaluso, E. (2010). Right temporal-parietal junction engagement during spatial reorienting does not depend on strategic attention control Neuropsychologia 48, 1160-1164.

Naumann, E., Bartussek, D., Diedrich, O., and Laufer, M. E. (1992). Assessing cognitive and affective information-processing functions of the brain by means of the late positive complex of the eventrelated potential. J. Psychophysiol. 6, 285-298.

Ohman, A., Flykt, A., and Esteves, F. (2001). Emotion drives attention: detecting the snake in the grass. J. Exp. Psychol. Gen. 130, 466-478.

Phelps, E. A., Ling, S., and Carrasco, M. (2006). Emotion facilitates perception and potentiates the perceptual benefits of attention. Psychol. Sci. 17, 292-299.

Polich, J. (2007). Updating P300, an integrative theory of $\mathrm{P} 3 \mathrm{a}$ and $\mathrm{P} 3 \mathrm{~b}$. Clin. Neurophysiol. 118, 2128-2148.

Posner, M. I. (1980). Orienting of attention. Q. J. Exp. Psychol. 32, 3-25.

Pourtois, G., Grandjean, D., Sander, D., and Vuilleumier, P. (2004) Electrophysiological correlates of rapid spatial orienting towards fearful faces. Cereb. Cortex 14, 619-633.

Pourtois, G., Schettino, A., and Vuilleumier, P. (2012). Brain mechanisms for emotional influences on perception and attention: what is magic and what is not. Biol. Psychol. doi: 10.1016/j.biopsycho. 2012.02.007. [Epub ahead of print].

Pourtois, G., Schwartz, S., Seghier, M L., Lazeyras, F., and Vuilleumier, P. (2006). Neural systems for orienting attention to the location of threat signals: an event-related fMRI study. Neuroimage 31, 920-933.

Rosen, A. C., Rao, S. M., Caffarra, P., Scaglioni, A., Bobholz, J. A., Woodley, S. J., Hammeke, T. A., Cunningham, J. M., Prieto, T. E., and Binder, J. R. (1999). Neural basis of endogenous and exogenous spatial orienting: a functional MRI study. J. Cogn. Neurosci. 11, 135-152.

Sabatinelli, D., Lang, P. J., Bradley, M. M., Costa, V. D., and Keil, A. (2009) The timing of emotional discrimination in human amygdala and ventral visual cortex. J. Neurosci. 29, 14864-14868.

Schupp, H., Flaisch, T., Stockburger, J., and Junghofer, M. (2006). Emotion and attention: event-related brain potential studies. J. Psychophysiol. 20, 115.

Serences, J. T., Shomstein, S., Leber, A. B., Golay, X., Egeth, H. E., and Yantis, S. (2005). Coordination of voluntary and stimulus-driven attentional control in human cortex. Psychol. Sci. 16, 114-122.

Shafer, A. T., Matveychuk, D., Penney, T., O’Hare, A. J., Stokes, J., and Dolcos, F. (2012). Processing of emotional distraction is both automatic and modulated by attention: evidence from an eventrelated fMRI investigation. J. Cogn. Neurosci. 24, 1233-1252.

Vossel, S., Weidner, R., Thiel, C. M. and Fink, G. R. (2009). What is "Odd" in Posner's location-cueing paradigm? Neural responses to unexpected location and feature changes compared. J. Cogn. Neurosci. 21, 30-41.

Conflict of Interest Statement: The authors declare that the research was conducted in the absence of any commercial or financial relationships that could be construed as a potential conflict of interest.

Received: 14 February 2012; accepted: 16 April 2012; published online: 01 May 2012.

Citation: Frank DW and Sabatinelli D (2012) Stimulus-driven reorienting in the ventral frontoparietal attention network: the role of emotional content. Front. Hum. Neurosci. 6:116. doi: 10.3389/fnhum.2012.00116

Copyright (c) 2012 Frank and Sabatinelli. This is an open-access article distributed under the terms of the Creative Commons Attribution Non Commercial License, which permits non-commercial use, distribution, and reproduction in other forums, provided the original authors and source are credited. 